City University of New York (CUNY)

CUNY Academic Works

Publications and Research

Kingsborough Community College

2013

\title{
The Use of Statistics in Experimental Physics
}

Thomas J. Pfaff

Ithaca College

Maksim Sipos

University of Illinois at Urbana-Champaign

M. C. Sullivan

Ithaca College

B. G. Thompson

Ithaca College

Max Tran

CUNY Kingsborough Community College

\section{How does access to this work benefit you? Let us know!}

More information about this work at: https://academicworks.cuny.edu/kb_pubs/166

Discover additional works at: https://academicworks.cuny.edu

This work is made publicly available by the City University of New York (CUNY).

Contact: AcademicWorks@cuny.edu 


\title{
The Use of Statistics in Experimental Physics
}

\author{
Thomas J. Pfaff \\ Department of Mathematics \\ Ithaca College \\ Ithaca, NY 14850 \\ tpfaff@ithaca.edu \\ Maksim Sipos \\ Department of Physics \\ University of Illinois Urbana-Champaign \\ Urbana, IL 61801 \\ sipos2@illinois.edu \\ M. C. Sullivan \\ Department of Physics \\ Ithaca College \\ Ithaca, NY 14850 \\ mcsullivan@ithaca.edu \\ B. G. Thompson \\ Department of Physics \\ Ithaca College \\ Ithaca, NY 14850 \\ bthompso@ithaca.edu \\ Max M. Tran \\ Kingsborough Community College \\ Department of Math and Computer Science \\ 2001 Oriental Boulevard \\ Brooklyn, NY 11235 \\ mtran@kingsborough.edu
}


When we teach statistics courses, the examples we use are typically slanted to the biological and health sciences or business and economics. We don't usually think of physics, despite the fact that statistics and probability are used a extensively in physics. For example, in statistical mechanics, physicists use statistical tools to analyze behavior of systems with many degrees of freedom, and in quantum mechanics, physicists calculate probabilities of observing an outcome of a measurement. The use of statistics in uncertainty analysis and data analysis has long been a tool used by physicists. Some of the methods used can be quite complex, such as extracting random and systematic errors in the experiment from curve fits [2] while other examples from nuclear physics are more straightforward [3].

Even straightforward tasks such as residuals analysis, can be quite rewarding in the information that can be derived from seemingly simple data. For example, examining the residuals from a Fourier analysis of tidal motion can yield a more precise power spectrum [7]. In another example, comparing the measured flow of a viscous liquid to a model fit gives non-random residuals, which in turn suggests a way to improve the model to better describe the flow [4]. In a similar example a residual plot is particularly important part in an experiment in modeling rotational motion presented in $[12]$.

In this paper, we present three interesting examples in detail that make use of statistics in experimental physics, each of which could be used as an extended example or a case study in a statistics course. The first example looks at modeling angular velocity linearly. Here a residual plot shows that a linear model isn't the best model. In the next experiment, students see the use of residuals in an historically significant setting related to the quantum nature of radiation. This was used in a course for introductory physics majors as an exercise in data analysis using spreadsheets or Matlab. Finally, goodness of fit is used to examine data from a nuclear decay experiment, one of the cornerstones of modern quantum physics and a triumph of 20th century physics. In this experiment, the normal or gaussian distribution is used to check the accuracy, or lack thereof, of new equipment which was used in a junior-level laboratory course. 


\section{Drag on Rotational Motion}

In this experiment a cylindrical aluminum disk is mounted on an air bearing, which reduces the friction on the disk to very small levels by pushing air under the disk so that it is suspended by the air. The air has two competing effects on the spin of the disk. The first is due to the air rushing past small non-uniformities on the surface, which imparts a turbine torque that speeds up the rotation rate. The second is a small a small air friction that acts to slow it down. If the disk is set in place and left for a long time, it will spin up or down until it reaches a terminal velocity, which we designate $\omega_{\infty}$.

To begin the experiment the disk is spun up by hand to an initial angular velocity $\omega_{0}$ (in units of angle traced per unit time, $\mathrm{rad} / \mathrm{s}$ ). Then the angular velocity $\omega$ is measured as a function of time as the disc gradually slows down due to frictional drag. The goal is to specify an accurate mathematical model for the recorded motion, that is, to obtain only random fluctuations in the difference between the data and the model (the residuals). All the details and graphs of this experiment are available in a separate publication [12].

The mathematical model for a physical system that experiences drag due to air friction can be quite complicated in detail. However, it has be found that good fits can be obtained with models that have linear and quadratic terms in the velocity or angular velocity. The model for the time derivative of the angular velocity of the disk, $\omega^{\prime}$, up to second order in angular velocity ican be written as

$$
\omega^{\prime}=c_{T}-c_{1} \omega-c_{2} \omega 2
$$

where $c_{T}$ is the turbine torque and $c_{1}$ and $c_{2}$ are the linear and quadratic drag coefficients, all normalized to the disc's moment of inertia. Here, $c_{T}, c_{1}$ and $c_{2}$ all depend in some undetermined way on the pressure $p$ in the air bearing (in units of psi). In the theoretical model for the equation, it has been assumed that $\omega$ is always positive and that the turbine term $c_{T}$ is in the direction of $\omega$. Experimentally, an initial spin in the direction of the terminal angular velocity will ensure that the angular velocity does not go negative. In the region of low angular speed, the experiment can be modeled to first order, i.e., quadratic drag can be neglected. The above equation then reduces 
to $\omega^{\prime}=c_{T}-c_{1} \omega$ and has a closed form solution of

$$
\omega-\omega_{\infty}=\left(\omega_{0}-\omega_{\infty}\right) e^{-c_{1} t}
$$

By taking logarithm, equation (2) can be written in a linear form and a least-squares fitting can be applied to obtain estimates of the parameters $c_{T}$ and $c_{1}$.

While this model provides a good fit to the data, a plot of the residuals reveals a slight nonrandom discrepancy at early times. Early times correspond to higher angular speed where the term which is quadratic in the angular speed is expected have more of an effect and so the second order equation may be a better model. When the quadratic drag is present, (1) has the closed form solution

$$
\omega=\frac{\omega_{\infty}^{\prime}+\left(\omega_{\infty}^{\prime}+c_{1} / c_{2}\right) g\left(\omega_{0}\right) e^{-s t}}{1-g\left(\omega_{0}\right) e^{-s t}},
$$

where $g(\omega)=\left(\omega-\omega_{\infty}^{\prime}\right) /\left(\omega+\omega_{\infty}^{\prime}+c_{1} / c_{2}\right)$ and $\omega^{\prime}=c_{1} / 2 c_{2}\left(\sqrt{1+4 c_{T} c_{2} / c_{1} 2}-1\right)$. The nonlinear form of (3) resists linearization. Since there are three co-dependent fitting parameters, $c_{T}, c_{1}$ and $c_{2}$, it is difficult (but not impossible) to manually fit the model to the experimental data. As an alternative, we can use a computer method (e.g. fminsearch in Matlab ${ }^{\mathrm{TM}}$ ), to perform a non-linear automatic fitting and parameter estimation. The plot in Fig. 1 shows the values obtained for $c_{2}$ for several different air bearing pressures that were obtained using an automated three parameter fit. There is general agreement between the values obtained by fitting by hand and those obtained by using the automated method.

In addition to the reduction of workload that results from using an automated fitting method, it also gives estimates of the error in the fitted parameters. However, in this experiment, multiple runs of the experiment for the same values of the air bearing pressure, show that the estimated errors of $c_{2}$ calculated from the fit (red bars in Fig. 1) are smaller than the actual errors estimated from experimental data (blue bars in Fig. 1). Here, the additional error may be due to fluctuations in pressure in the air bearing and in the measurement of $\omega$. These sources of error were not reflected in the statistical model used in fminsearch, so, in this case, it is not justified to simply use the statistical error in determining the uncertainty in the value of $c_{2}$.

Overall, the experimental data are easily obtained and the simple linear model provides a good 


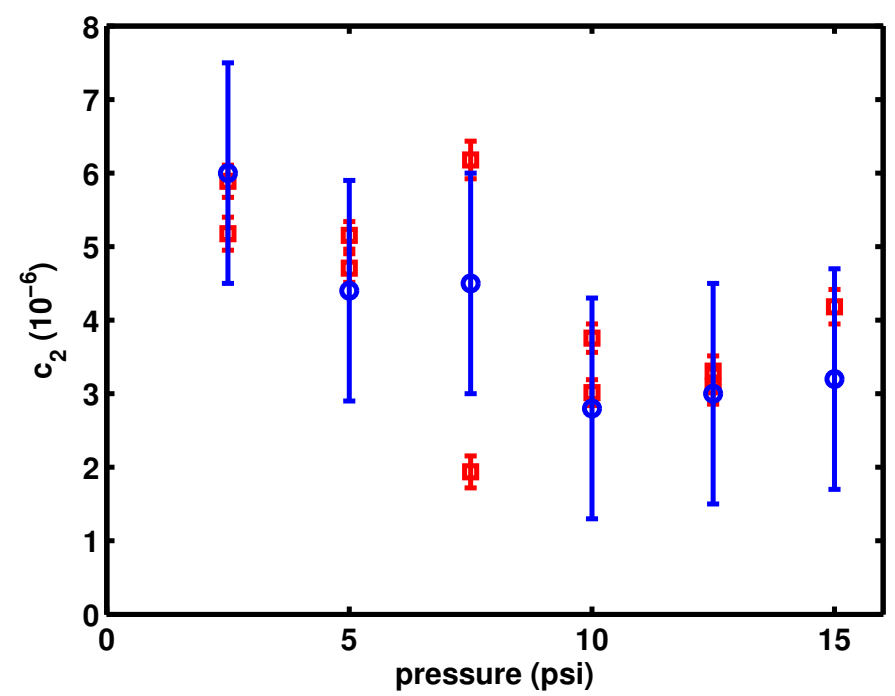

Figure 1: Values of $c_{2}$ computed from the fit of measured angular velocities to (3). Red error bars show errors computed by the numerical fitting procedure. Blue bars indicate errors estimated on the basis of unknowns in the experimental conditions.

fit. Yet the slight non-random discrepancy between the linear model and the data shows up in a plot of the residuals, thus emphasizing their value in fitting models to experimental data. Once a quadratic model is used, the residuals no longer show a pattern demonstrating that linear and quadratic drag are simultaneously active in the system. Further, this experiment demonstrates that one cannot always use the statistical quality-of-fit estimates to find error bars of experimental measurements. Instead one needs to keep in mind the global picture of the experiment, and what experimental conditions may make error bars larger.

\section{Taking the Average Temperature of the Universe}

We will now use a theoretical thermometer to measure the average temperature of the universe by fitting a model, which has one parameter, to data. As in many such examples we want to estimate the parameter, and also assess how well the model fits the data using residual. But first a brief overview of the physics involved.

One of the crowning achievements of modern physics was the discovery of the quantum nature 
of radiation by Max Planck in 1900 [9]. Any object at temperatures above absolute zero will emit electromagnetic radiation, or light. If the object absorbs light perfectly, then it will also be a perfect emitter of this thermal radiation. Black objects are reasonably close to perfect absorbers of light, which makes black objects - "a blackbody" - also a perfect emitter. A good example of a blackbody in everyday life would be a red-hot stove. Most of the radiation the red-hot stove emits is not in the visible spectrum, rather in the infrared, but its emissions are so intense we can feel the radiation.

Planck was trying to understand the intensity vs. frequency behavior of blackbody radiation, in other words, the spectrum of light from a hot body. Expressions derived from classical physics were unable to predict the shape of the laboratory data. By introducing the concept that electromagnetic radiation (light and radio waves) behaved as if composed of bundles of energy (photons), he derived a formula which did fit the data. This was the start of the quantum revolution in physics.

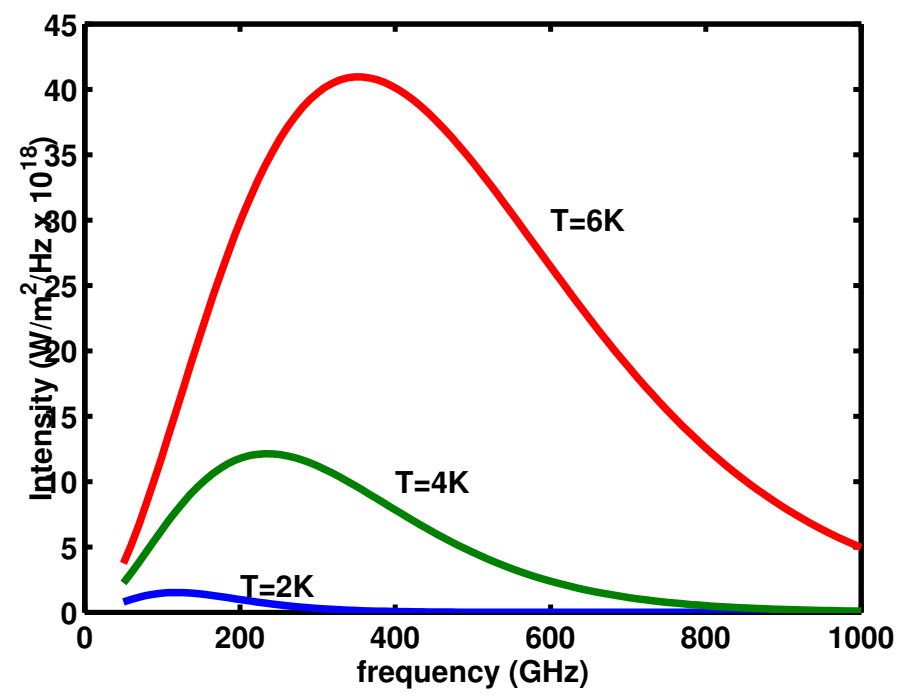

Figure 2: A plot of the intensity of the radiation of a blackbody vs. frequency for temperatures of 2, 4 and 6 Kelvin.

Planck's blackbody radiation equation is as follows:

$$
I(f)=\frac{2 h f^{3}}{c^{2}} \frac{1}{e^{h f / k T}-1}
$$

where $I$ is the intensity of the radiation $\left(\mathrm{W} / \mathrm{m}^{2} / \mathrm{Hz}\right), f$ is the frequency of the radiation, $h$ is Planck's constant $\left(6.626069 \times 10^{-34} \mathrm{~J}-\mathrm{s}\right), c$ is the speed of light $\left(2.99792458 \times 10^{8} \mathrm{~m} / \mathrm{s}\right), k$ is the 
Boltzmann's constant $\left(1.38065 \times 10^{-23} \mathrm{~J} / \mathrm{K}\right)$, and $T$ is the absolute temperature of the blackbody in Kelvin. Notice that the only adjustable parameter in the formula is $T$, the temperature of the object. All the other factors are constants. In Fig. 2, we see a plot of $I$ vs. $f$ for several different values of the temperature and see that as the temperature increases, the peak intensity and peak frequency increase.

One of the crowning achievements of astronomy is the discovery that the universe began as a very compact fireball (the Big Bang) 13.75 billion years ago [10]. Because the universe is so large, it behaves as a single object at a uniform temperature, which makes the universe a huge blackbody that has been expanding and cooling ever since. In 1948, Alpher and Hermann estimated that the present average temperature of the universe is about $5 \mathrm{~K}$ [1]. Then in 1964, Penzias and Wilson, two researchers at Bell Labs, discovered a source of noise in their radio antennas that they couldn’t attribute to any earthly source [8]. Planck showed that blackbodies of various temperatures emitted radiation at certain frequencies, and Penzias and Wilson were able to relate the frequencies they measured to a blackbody of very low temperature, roughly $3 \mathrm{~K}$. Because the noise they measured came from all directions equally, there was no directed source for the radiation. This meant the radiation came from outside the solar system, and indeed, outside our galaxy! Penzias and Wilson quickly realized that they were measuring radiation emitted by the entire universe as predicted by Alpher and Hermann. The noise they measured was blackbody radiation left from the Big Bang, now called the cosmic microwave background radiation (CMB). In 1989, NASA launched the Cosmic Background Explorer (COBE) spacecraft, whose mission was to make detailed measurements of the CMB [5].

Figure 3 is an intensity vs. frequency plot of data from COBE [6] together with a best fit blackbody model using a temperature of $2.725 \mathrm{~K}$. The uncertainties in the data points are not visible as they are less than the width of the line. The remarkable agreement between these data and the model is obvious. More astounding is that this measurement of the universe as a whole can be characterized to a high degree of accuracy by a single number, the average temperature.

A plot of the residuals for the fit when $T=2.725 \mathrm{~K}$, the blue stars in Figure 4, shows that the remaining discrepancies are random and are of a magnitude which is the same as the uncertainty 


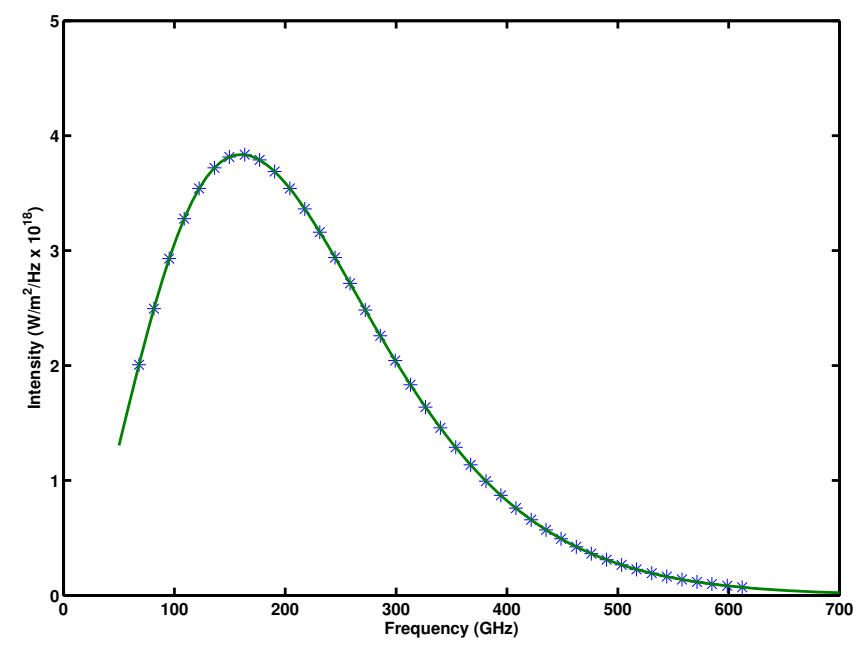

Figure 3: A plot of the microwave intensity vs. frequency data from the COBE spacecraft (dots) together with a blackbody model with a temperature of $2.725 \mathrm{~K}$ (line). The uncertainty in the data measurements is approximately $2 \times 10^{-22} \mathrm{~W} / \mathrm{m}^{2} / H z$ and thus is much smaller than the width of the line on the plot.

in the data themselves. The rms (root mean square) deviation from the model fit is at the level of $50 \mathrm{ppm}$ of the peak intensity. An informative exercise is to change the model temperature and observe the change in the residual plot. Even a $0.001 \mathrm{~K}$ change - higher or lower - leads to residuals that show systematic discrepancies in the fit (Figure 4, the green circles or the red squares) even though the Intensity plot is indistinguishable from the one shown in Figure 3.

We give our students this project of fitting these data with the blackbody model by trial and error, making sure that they produce a plot of the residuals in addition to the intensity plot and a calculation of the rms deviation. They immediately see how sensitive it is to small temperature changes and are able to home in on the average temperature of the universe quickly.

\section{Nuclear Counting of Cesium-137}

Nuclear counting experiments are a staple of all instructional physics laboratories. These atomic decays occur spontaneously and randomly. In a common laboratory experiment, one of the neutrons 


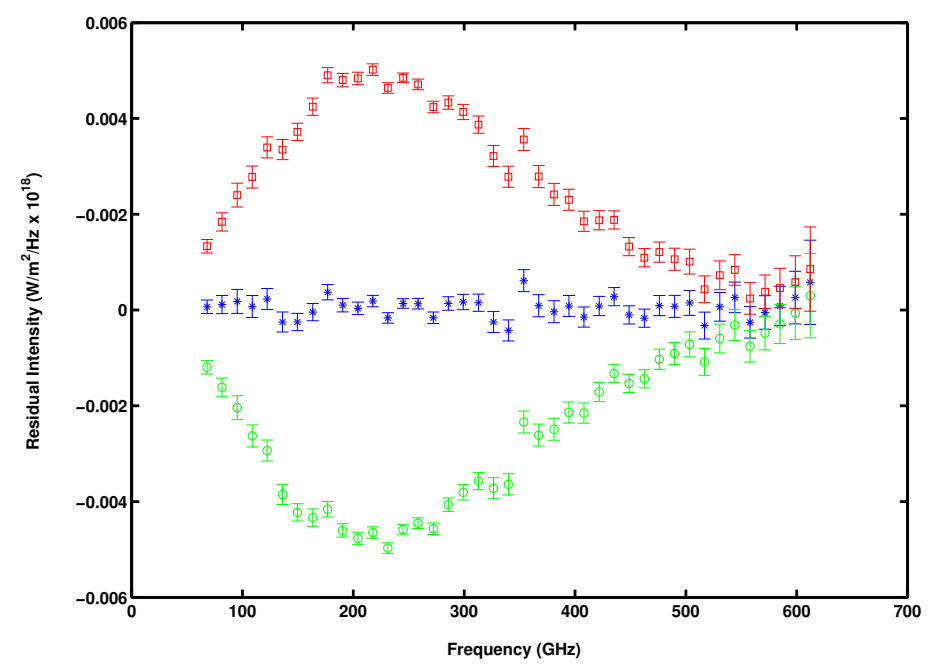

Figure 4: A plot of the residuals obtained by subtracting the model fit from the data in Figure 3. The uncertainties $(1 \sigma)$ in the intensities measured by COBE are also plotted and are of a magnitude of the model discrepancies. The fit for $T=2.725 \mathrm{~K}$ (blue stars) shows no systematic differences, just random ones. The rms deviation of the fit is $50 \mathrm{ppm}$ of the peak intensity. However, a plot of the residuals obtained when the model temperature is changed by $0.001 \mathrm{~K}$ to $2.724 \mathrm{~K}$ (red squares) or $2.726 \mathrm{~K}$ (green circles) shows that there is a systematic difference between the model and data.

inside ${ }^{137} \mathrm{Cs}$ spontaneously decays, becoming a proton, and ${ }^{137} \mathrm{Cs}$ becomes ${ }^{137} \mathrm{Ba}$. When this decay occurs, the atom emits an electron, an anti-neutrino, and a high-energy gamma ray. Of the byproducts of this nuclear decay, the gamma rays are the easiest to detect.

Students of mathematics are familiar with the fact that the rate of these spontaneous nuclear transformations is not constant and decreases with time. These transformations occur less and less frequently as time goes on, and follow an exponential decay. The decay constant is measured by the half-life; that is, the time required for half of the original material (in our case, ${ }^{137} \mathrm{Cs}$ ) to decay into the product $\left({ }^{137} \mathrm{Ba}\right)$.

However, if the half-life is long, then on the time scale of an undergraduate laboratory experiment, the rate at which the nuclei spontaneously transform can be considered constant. Thus, studying the rate at which gamma rays are emitted from a sample of ${ }^{137} \mathrm{Cs}$ is an ideal example of a Poisson process in time. A Poisson process is an experiment in which events occur at random 
times which are uniform over an interval and independent of each other - which exactly describes nuclear decays. The process is characterized by one parameter, the rate, or the expected number of events per unit time. The rate has units of time ${ }^{-1}$.

If used in a mathematics classroom, nuclear decay data can demonstrate the power of statistics for verifying physical data. A useful example of the use of nuclear decays in a statistics classroom is to take nuclear decay data and analyze subsets of the data, showing that for more and more data points, the fit to a bell curve becomes better and better.[?] In what follows, nuclear decay can also be used as an example of the differences between Gaussian and Poisson distributions.

In the physical experiment, we place an ampule of ${ }^{137} \mathrm{Cs}$ a few centimeters from a scintillation detector. Every time a gamma ray is incident on the scintillating medium the detector will convert the gamma ray into a voltage pulse with a peaked shape. This voltage pulse is then amplified and put through a linear gate and stretcher. The linear gate and stretcher converts the voltage pulses into square-wave pulses of given width (user-definable, between 0.5 and $5 \mu \mathrm{s}$ ). This process makes the pulses easier to detect with a computer. The square-wave output of the linear gate and stretcher is routed to the computerized data acquisition hardware. The goal is to convert each nuclear decay into a count registered by the computer. If this occurs, then we should expect a Poisson distribution of counts - and if we do not see a Poisson distribution, then we have to search for the reason why.

To count the pulses, we have long used a microcomputer designed and built by one of our faculty members specifically for this experiment, nicknamed "Femto." In 2006, we purchased a new data acquisition card commercially available from National Instruments, the PCI-MIO-16E4, and use a simple MatLab routine to collect the data from the acquisition card. To address the question of whether or not the National Instruments data acquisition system can replace Femto, we would be wise to see if the data acquisition card can also accurately record the voltage pulses. Thus, we performed an experiment to compare our two data acquisition systems. The experiment consisted of placing a radioactive source a certain distance away from the scintillation detector. We then counted the number of decays in 16,400 periods of 0.1 s each, fitting both the Poisson distribution and the Gaussian distribution to the data.

In the experiment, we have $n$ radioactive atoms, each with a certain probability $p$ of decaying 
and being counted in a given time interval, and a probability of $r$ counts during this time interval. Typically, we have $n$ on the order of Avogadro's number, $n>10^{25}, p \approx 10^{-20}$, giving an average number of decays $n p \equiv \mu \approx 10^{5}$. However, the gamma rays emitted as a by-product of the radioactive decay are emitted in all directions. Our experimental detector can only detect gamma rays emitted directly towards our detector, so for these reasons, $r \approx 100$. Since $n$ is large, we have $r \ll n$. This means that only a very small proportion of the atoms decay in each time-slice. When these conditions are met then the distribution is closely approximated by the Poisson distribution given by:

$$
p_{\text {Poisson }}(r)=\frac{(\mu)^{r}}{r !} e^{-\mu}
$$

While we do not know $n$ or $p$, we can approximate the true mean $\mu$ by the average $\bar{r}$, where $\bar{r}$ can be calculated from the data. Taking $\mu \approx \bar{r}$, we can fit the expected distribution $p_{\text {Poisson }}(r)$ to our data. We perform this fit setting the parameter $\mu=\bar{r}$.

When $r, n$, and $n-r$ are all large, the Poisson distribution approaches the Gaussian distribution, given by:

$$
\begin{aligned}
p_{\text {Gaussian }}(r) & =\frac{1}{\sqrt{2 \pi \sigma^{2}}} e^{-(r-\mu)^{2} / 2 \sigma^{2}} \\
& =\frac{1}{\sqrt{2 \pi n p}} e^{-(r-\mu)^{2} / 2 n p}
\end{aligned}
$$

It is a good exercise for math students to show that for the Poisson distribution, the width of the distribution is the square root of the mean, therefore $\sigma=\sqrt{\mu}$. This allows us to substitute $\sigma=\sqrt{n p}$ in (6). If we again find $\bar{r}$, then we can take $\mu \equiv n p \approx \bar{r}$ and fit our data using the Gaussian distribution. The Gaussian fit is performed by setting $\mu=\bar{r}$ and $\sigma$ to the standard deviation calculated from the data. The Gaussian distribution has another useful prediction: In a Gaussian distribution, we expect $68 \%$ of the data to lie within $\mu \pm \sigma$ and $95.4 \%$ of the data to lie within $\mu \pm 2 \sigma$.

Finally, we can test the goodness of our fits. A statistic often used to verify a quality of a fit, is the " $\chi^{2}$ test". For our problem, " $\chi^{2}$ per degree of freedom" is defined as

$$
\chi_{\nu}^{2}=\frac{1}{\nu} \sum_{r} \frac{\left(p_{t h}(r)-p_{\exp }(r)\right)^{2}}{p_{t h}(r)}
$$


where $p(r)$ is the theoretical or experimental probability distribution and $\nu$ is the number of degrees of freedom in the fit, equal to the number of different values of $r$ minus the number of adjustable parameters in the fitting function. $\nu \approx 100$ for both of our datasets. As the quality of the fit increases, we expect the numerator to become small, thus we expect the $\chi^{2}$ statistic to become smaller with a better quality of fit.

The data from the experiment using the home-built microcomputer Femto is shown in Fig. 5. In our experiment, $n, r$, and $n-r$ are large, so the radioactive decay data should fit the requirements of the Poisson and the Gaussian distributions, and indeed, we see excellent agreement between the fits and the experimental data. The mean was found to be $\bar{r}=154.2 \pm 0.1$ counts per 0.1 s with a standard deviation of $\sigma=12.2 \pm 0.1$ counts per $\sqrt{0.1 \mathrm{~s}}$. The goodness of fit as measured by $\chi^{2}$ is similarly reasonable for both the Poisson and Gaussian fits. Moreover, we expect $\sigma=\sqrt{\bar{r}}$, and we find that $\sqrt{\bar{r}}=12.4$ counts per $\sqrt{0.1 \mathrm{~s}}$, in good agreement with predictions. Finally, the data also obey what we expect from the Gaussian distribution: $69 \%$ of the data lie within $\mu \pm \sqrt{\bar{r}}$ (predicted: $68 \%$ ), and $96 \%$ lie within $\mu \pm 2 \sqrt{\bar{r}}$ (predicted: 95.4\%). These results are collected in Table 1.

The data from the National Instruments card and MatLab, shown in Fig. 6 is markedly different. We are unable to obtain good fits using the Poisson distribution. However, we can still get good fits (as measured by the goodness-of-fit parameter) for the Gaussian distribution. At this stage, a student might be tempted to throw away the Poisson fit and accept the results of the Gaussian fit. However, on closer inspection, we even see that the data do not behave as the good Gaussian fit predicts. As discussed above, counting experiments (such as radioactive decays), because they occur randomly but at a certain rate, should obey the Poisson distribution, and thus have a standard deviation where $\sigma=\sqrt{\bar{r}}$. The standard deviation in this experiment is $15.3 \pm 0.1$ per $\sqrt{0.1 \mathrm{~s}}$, whereas $\sqrt{\bar{r}}=11.8$, significantly smaller than we would expect. This carries through to the rest of the distribution: we only find $56 \%$ of the data lies between $\mu \pm \sqrt{\bar{r}}$ (predicted: $68 \%$ ), and only $88 \%$ lies between $\mu \pm 2 \sqrt{\bar{r}}$ (predicted: $95.4 \%$ ).

This closer inspection indicates that there is additional error besides counting. Because the experiments were identical except for the computerized data acquisition systems, clearly the error must come from the National Instruments PCI-MIO-16E4 data acquisition card or the MatLab 


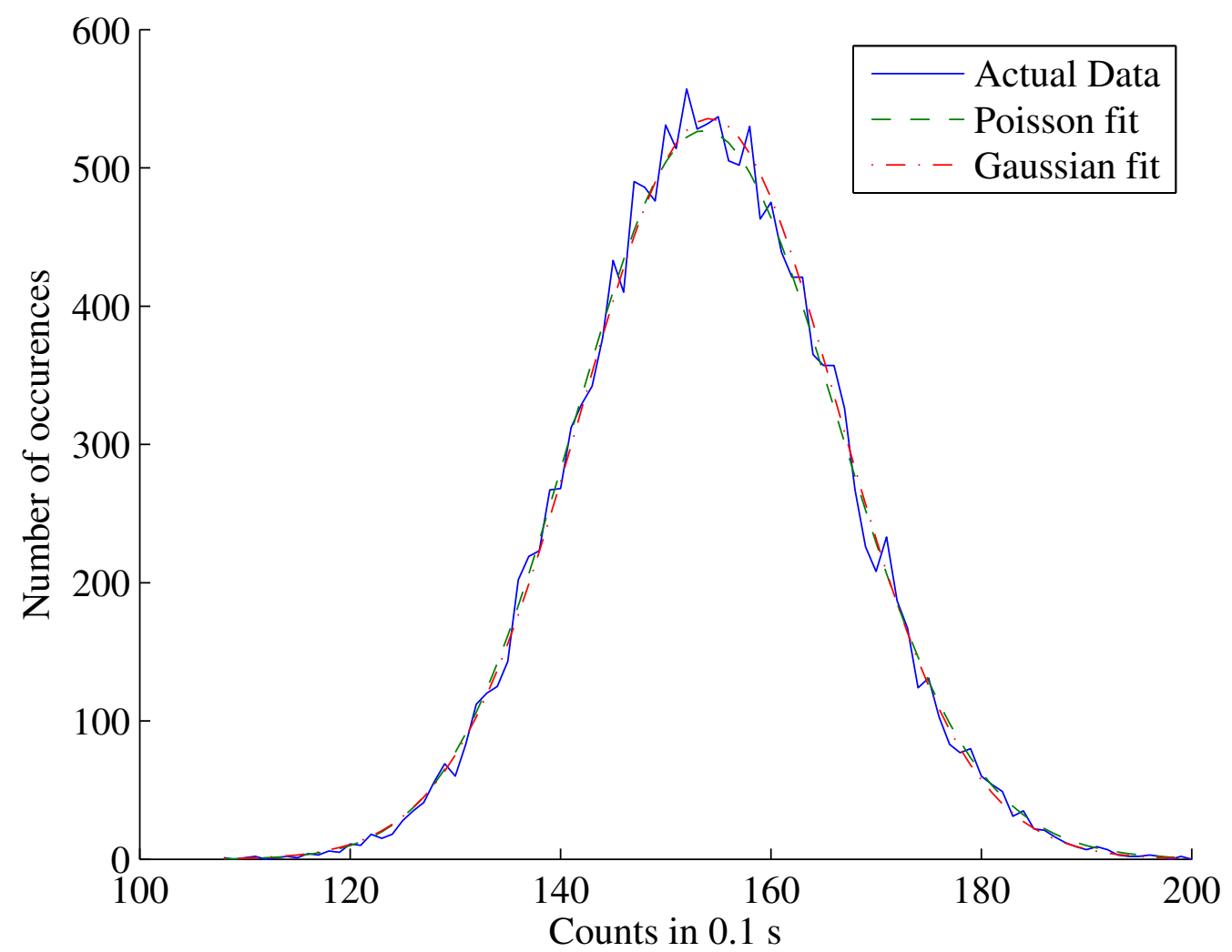

Figure 5: Histogram of the 16,400 decay count measurements using our home-built microcomputer "Femto". The mean was found to be $\bar{r}=154.2 \pm 0.1$ per $0.1 \mathrm{~s}$. The standard deviation of the distribution was found to be $\sigma=12.2$ per 0.1s. Gaussian (dot-dashed line) and Poisson (dashed) fits show excellent agreement between the data and the model. Analysis of the fits can be seen in Table 1. 


\begin{tabular}{|l|c|c|}
\hline & Microcomputer Femto & National Instruments PCI-MIO-16E4 \& MatLab \\
\hline $\bar{r}$ & $154.2 \pm 0.1$ per $0.1 \mathrm{~s}$ & $140.4 \pm 0.1$ per $0.1 \mathrm{~s}$ \\
\hline$\sigma$ & $12.2 \operatorname{per} \sqrt{0.1 \mathrm{~s}}$ & 15.3 per $\sqrt{0.1 \mathrm{~s}}$ \\
\hline$\sqrt{\bar{r}}$ & $12.4 \operatorname{per} \sqrt{0.1 \mathrm{~s}}$ & 11.8 per $\sqrt{0.1 \mathrm{~s}}$ \\
\hline$\chi_{P}^{2}$ & 0.85 & 64.7 \\
\hline$\chi_{G}^{2}$ & 1.06 & 1.71 \\
\hline $\bar{r} \pm \sqrt{\bar{r}}$ & $69 \%$ & $56 \%$ \\
\hline $\bar{r} \pm 2 \sqrt{\bar{r}}$ & $96 \%$ & $88 \%$ \\
\hline
\end{tabular}

Table 1: Comparison of the fits computed from the datasets for the National Instruments PCI-MIO16E4 data acquisition card and the microcomputer Femto. The error bars for $\bar{r}$ are the standard deviation of the mean. $\sigma$ is the standard deviation, and we expect $\sigma=\sqrt{\bar{r}} . \chi_{P}^{2}$ and $\chi_{G}^{2}$ are the values of the goodness-of-fit parameter for Poisson and Gaussian distribution fits, respectively. $\bar{r} \pm \sqrt{\bar{r}}$ and $\bar{r} \pm 2 \sqrt{\bar{r}}$ represent the fraction of the data in the stated range. We get uniformly good fits using Femto, and a good Gaussian fit using the PCI-MIO-16E4 card, though less data is within $\bar{r} \pm 2 \sqrt{\bar{r}}$ than is predicted from the Gaussian distribution. 


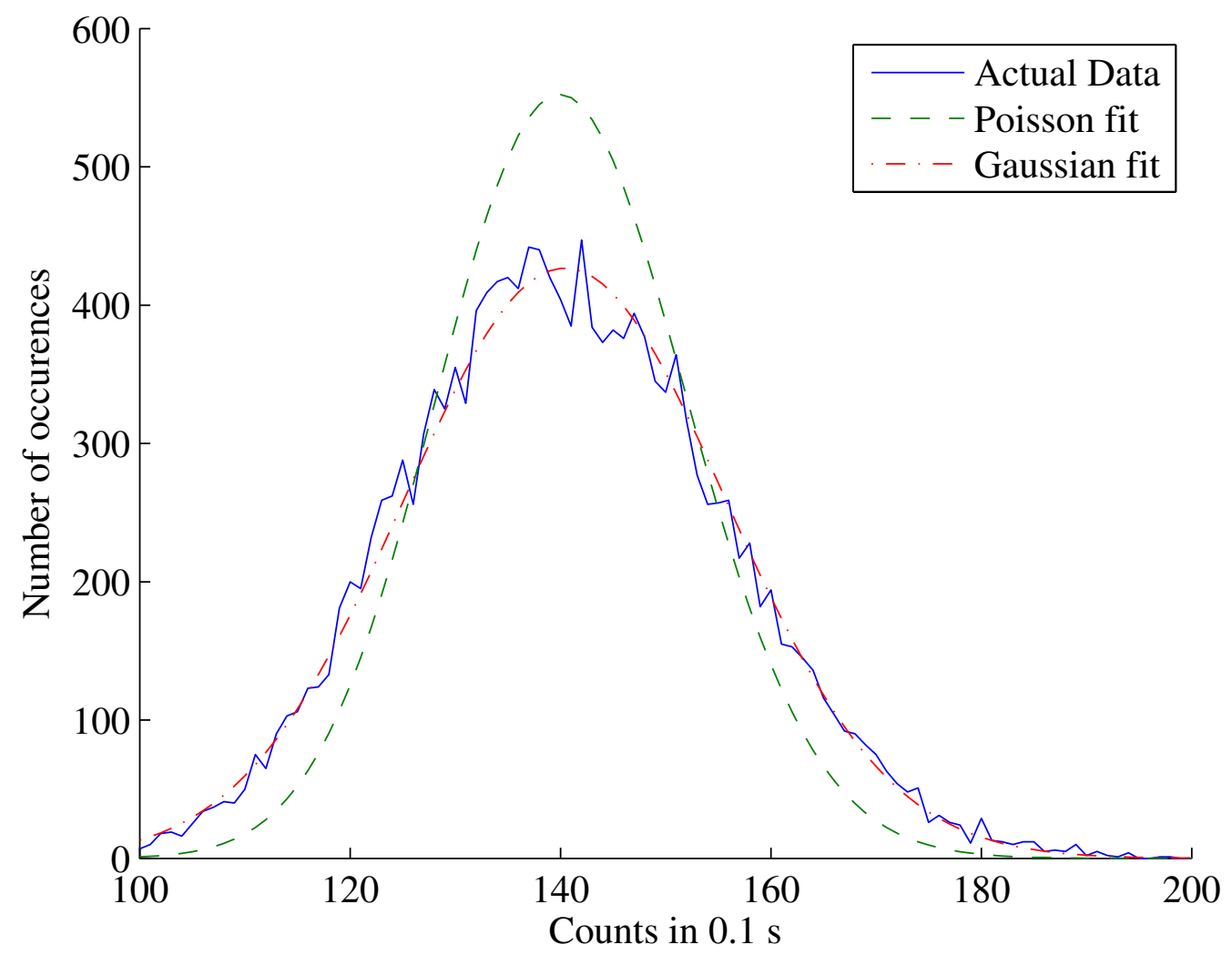

Figure 6: Histogram of the 16,400 decay count measurements using the National Instruments PCIMIO-16E4 data acquisition card and a simple MatLab routine. The mean was found to be $\bar{r}=$ $140.4 \pm 0.1$. The standard deviation of the distribution was found to be $15.3 \pm 0.1$ per $\sqrt{0.1 \mathrm{~s}}$. The Gaussian (dot-dashed line) fit show excellent agreement between the data and the model, however the Poisson (dashed) fit shows poor agreement. Analysis of the fits can be seen in Table 1. 
routine used to collect the data from the card. The additional error results in missed counts. These missed counts can be caused by two sources. They can occur when two pulses are too close to each other and are detected as one by the sampling hardware. Or, if pulses arrive during the time when the MatLab routine is converting analog voltage to a digital signal, those pulses are lost. The pulses counted had a width of $5 \mu \mathrm{s}$, which corresponds to a frequency of $200 \mathrm{KHz}$. The NI card's maximum sampling rate is $250 \mathrm{KHz}$, which is sufficient to count nearly all of the pulses. Thus, the counting error comes from the MatLab routine. Our simple routine continually receives and converts voltages from analog to digital, resulting in many lost pulses when the computer is busy during the conversion. Because this error is random, and comes in addition to the intrinsic counting error, the result is a good fit of the Gaussian distribution but a poor fit of the Poisson distribution

to the data, as seen in Fig. 6. This also explains why we have recorded $\sigma \approx 15$ per $\sqrt{0.1 \mathrm{~s}}$ while $\sqrt{\bar{r}} \approx 12$ per $\sqrt{0.1 \mathrm{~s}}$. It is also clear that we are missing counts as the experimental setup did not change but the average counts per $0.1 \mathrm{~s}$ decreased by approximately 14 . Note that this error is somewhat fortuitous - had the error been systematic rather than random neither the Poisson nor the Gaussian distribution would have fit the data with a reasonable goodness-of-fit parameter.

Thus we used two different computerized data collection systems to analyze the voltage pulses coming from a radioactive decay experiment. Using our home-built microcomputer, we find data that agree well with statistical predictions. Using a commercially available data acquisition system, we get good fits to the data using a Gaussian distribution, and poor fits using Poisson distribution. The take-home lesson here for mathematics students is to consider what a careful mathematical analysis of the data implies. Rather than accepting the Gaussian fit, a careful analysis of the errors in the fit leads us to understand what is wrong with using the commercial data acquisition system and even an estimate of how many counts it misses in every $0.1 \mathrm{~s}$.

\section{Conclusion}

Statistical analysis plays a major role in several branches of physics, but it is also very useful for elementary physics. As the various experiments discussed in this paper illustrate, the use of statistical analysis especially in the form of residual analysis can help determine the best fit model 
for experimental data. In other cases, it can be used to pinpoint flaws in laboratory equipment.

Our goal in writing this paper was to provide mathematicians with these example. We do hope that everyone who read this enjoyed the connections between statistics and physics, but we also hope some of these examples find their way into statistics classrooms. This could happen in many ways. Instructors could simply take a few moments in class and discuss these examples or even provide students with this paper. In other cases, ambitious instructors could use the examples here to create assignments for students. In the end, keep physics in mind the next time you are in a statistics classroom.

\section{Acknowledgement}

The authors gratefully acknowledge Daniel A. Briotta Jr. for his amazing work and dedication to the task of undergraduate education displayed in his construction of the microcomputer "Femto."

\section{References}

[1] R. A. Alpher and R. C. Herman, Evolution of the Universe, Nature, 162 (1948) 774.

[2] J. Bechhoefer, Curve fits in the presence of random and systematic error, Am. J. Phys. 68 (2000) 424-429.

[3] Byron Curry, Dave Riggins, and P. B. Siegel, Data analysis in the undergraduate nuclear laboratory, Am. J. Phys. 63 (1995) 71.

[4] Rafael M. Digilov and M. Reiner, Weight-controlled capillary viscometer, Am. J. Phys. 73 (2005) 1020-1022.

[5] D. J. Fixsen, E. S. Cheng, J. M. Gales, J. C. Mather, R. A. ShaFer and E. L. Wright, The Cosmic Microwave Background spectrum from the full COBE FIRAS data set, Astrophysical Journal, 473 (1996) 576. 
[6] Legacy Archive for Microwave Background Data Analysis. http://lambda.gsfc.nasa.gov/product/cobe/firas_monopole_get.cfm

[7] Robert de Levie, Tidal analysis on a spreadsheet, Am. J. Phys. 72 (2004) 644-651.

[8] see Mather 1993 A. A. Penzias and R. W. Wilson, A measurement of the excess antenna temperature at 4080Mc/s, Astrophysical Journal, 142 (1965) 419-421.

[9] Max Planck, On the Law of Distribution of Energy in the Normal Spectrum, Annalen der Physik 4 (1901), 553.

[10] S. H. Suyu, P. J. Marshall, M. W. Auger, S. Hilbert, R. D. Blandford, L. V. E. Koopmans, C. D. Fassnacht and T. Treu, Dissecting the Gravitational Lens B1608+656, Astrophysical Journal, 711 (2010), 201-222.

[11] J. R. Taylor, An Introduction to Error Analysis, 2nd Edition, University Science Books, 1997.

[12] B.G. Thompson and P.A. Smith, An experiment in roational motion with linear and quadratic drag, Am. J. Phys. 72(4) (June 2004) 839-842. 